\title{
$\mathrm{BN} / \mathrm{SiC}$ 复合界面层对 $\mathrm{SiC}$ 纤维和 PIP-Mini 复合材料力学性能的影响
}

\author{
吕晓旭 ${ }^{1}$, 姜卓钰 ${ }^{1}$, 周怡然 ${ }^{1}$, 齐 哲 ${ }^{1}$, 赵文青 ${ }^{1,2}$, 焦 健 $^{1}$
}

(1. 中国航发北京航空材料研究院 先进复合材料国防科技重点实验室, 北京 100095 ; 2. 北京理工大学 材料学院, 北京 100081)

摘 要: 采用化学气相渗透(CVI)工艺, 在 $\mathrm{SiC}$ 纤维表面沉积 $\mathrm{BN}$ 和 $\mathrm{BN} / \mathrm{SiC}$ 复合界面层, 对沉积界面层前后纤维的 力学性能进行了评价。采用聚合物浸渍裂解(PIP)工艺进行致密化, 制得以原纤维、 $\mathrm{BN}$ 界面层和 $\mathrm{BN} / \mathrm{SiC}$ 界面层纤 维增强的三种 $\mathrm{Mini}-\mathrm{SiC}_{\mathrm{f}} / \mathrm{SiC}$ 复合材料, 研究其微观结构和拉伸性能。结果表明: 采用 CVI 工艺制得的界面层厚度 均匀、结构致密, 其中 $\mathrm{BN}$ 界面层中存在六方相, 晶体尺寸为 $1.76 \mathrm{~nm}$; $\mathrm{SiC}$ 界面层结晶性较好, 晶粒尺寸为 $18.73 \mathrm{~nm}$; 沉积界面层后 $\mathrm{SiC}$ 纤维的弹性模量基本保持不变, 拉伸强度降低。与 $\mathrm{SiC}_{\mathrm{f}} / \mathrm{SiC}$ 相比, $\mathrm{PIP}$ 工艺制备的 $\mathrm{SiC}_{\mathrm{f}} / \mathrm{BN} / \mathrm{SiC}$ 和 $\mathrm{SiC}_{\mathrm{f}} /(\mathrm{BN} / \mathrm{SiC}) / \mathrm{SiC}-\mathrm{Mini}$ 复合材料所能承受的最大拉伸载荷和断裂应变明显提升, $\mathrm{BN}$ 界面层起主要作用。由断面形 貌分析可以看出, $\mathrm{SiC}_{\mathrm{f}} / \mathrm{BN} / \mathrm{SiC}$ 和 $\mathrm{SiC}_{\mathrm{f}} /(\mathrm{BN} / \mathrm{SiC}) / \mathrm{SiC}$ 复合材料的纤维拔出明显, 说明在断裂时消耗的能量增加, 可承 受的最大载荷增大。

关 键 词: $\mathrm{BN} / \mathrm{SiC}$ 复合界面层; $\mathrm{Mini}_{-}-\mathrm{SiC}_{\mathrm{f}} / \mathrm{SiC}$ 复合材料; 最大拉伸载荷; 断裂应变

中图分类号: TB332 文献标识码: A

\section{Effect of BN/SiC Multilayered Interphases on Mechanical Properties of SiC Fibers and Minicomposites by PIP}

\author{
LÜ Xiaoxu ${ }^{1}$, JIANG Zhuyu ${ }^{1}$, ZHOU Yiran ${ }^{1}$, QI Zhe ${ }^{1}$, ZHAO Wenqing ${ }^{1,2}$, JIAO Jian ${ }^{1}$
}

(1. National Key Laboratory of Advanced Composites, AECC Beijing Institute of Aeronautical Materials, Beijing 100095, China; 2. School of Materials Science \& Engineering, Beijing Institute of Technology, Beijing 100081, China)

\begin{abstract}
BN}$ and $\mathrm{BN} / \mathrm{SiC}$ interphases were deposited on the surface of $\mathrm{SiC}$ fibers by $\mathrm{CVI}$ process, and the mechanical properties of the as-received and coated fibers were evaluated. $\mathrm{SiC}_{\mathrm{f}} / \mathrm{SiC}$ minicomposites were prepared by PIP using the as-received, BN-coated and $\mathrm{BN} / \mathrm{SiC}$ coated fiber bundles as reinforcements. The effects of interphases on the mechanical properties of the composites were studied. The results show that the interphases prepared by CVI process are uniform and compact. The deposited BN interphase contains hexagonal phases with small crystal size (1.76 nm). The deposited $\mathrm{SiC}$ interphase has better crystallinity and larger grain size $(18.73 \mathrm{~nm})$ than $\mathrm{BN}$ interphase. The elastic modulus of coated $\mathrm{SiC}$ fibers shows basically no change, but the tensile strength decreases. The maximum tensile load and fracture strain of $\mathrm{SiC}_{\mathrm{f}} / \mathrm{BN} / \mathrm{SiC}$ and $\mathrm{SiC}_{\mathrm{f}} /(\mathrm{BN} / \mathrm{SiC}) / \mathrm{SiC}$ minicomposites are significantly increased, in comparison to $\mathrm{SiC}_{\mathrm{f}} / \mathrm{SiC}$ minicomposites. It can be seen from the cross-sections of $\mathrm{SiC}_{\mathrm{f}} / \mathrm{BN} / \mathrm{SiC}$ and $\mathrm{SiC}_{\mathrm{f}} /(\mathrm{BN} / \mathrm{SiC}) / \mathrm{SiC}$ mini-composites that the fibers with interphases pull out obviously relative to $\mathrm{SiC}_{\mathrm{f}} / \mathrm{SiC}$ mini-composites, and the $\mathrm{BN}$
\end{abstract}

收稿日期：2019-12-23; 收到修改稿日期：2020-02-01

基金项目: 国家科技重大专项(2017-VI-0007-0077) National Science and Technology Major Project (2017-VI-0007-0077)

作者简介: 吕晓旭(1988-), 男, 博士. E-mail: xiaoxul@126.com LÜ Xiaoxu(1988-), male, PhD. E-mail: xiaoxul@126.com

通讯作者：焦 健，研究员. E-mail: jian.jiao@biam.ac.cn JIAO Jian, professor. E-mail: jian.jiao@biam.ac.cn 
interphases played a reinforcing role in the tensile fracture process of the composites. The composites with interphases exhibit obvious fiber pull-out resulting in more energy consumption during the fracture, so that the composite can endure more load.

Key words: $\mathrm{BN} / \mathrm{SiC}$ multilayered interphase; $\mathrm{Mini}_{-} \mathrm{SiC}_{\mathrm{f}} / \mathrm{SiC}$ composites; maximum tensile load; fracture strain

$\mathrm{SiC}_{\mathrm{f}} / \mathrm{SiC}$ 复合材料由基体、界面层和纤维组成, 因其低密度、抗氧化和抗蠕变等优点被广泛应用于 航空发动机等热端部件 ${ }^{[1-3]}$ 。在服役过程中, 复合材 料基体开裂形成横向裂纹, 为氧元素等提供通道, 从而降低材料的服役寿命。基体开裂受初始缺陷 数、界面性质和热残余应力的影响 ${ }^{[4-5]}$, 当载荷经基 体传递到纤维时，纤维和基体之间的界面层会产生 摩擦滑动, 因此界面层必须是低能裂纹扩展面, 使 基体裂纹在界面处发生偏转，从而提高材料的损伤 容限 ${ }^{[6]}$ 。

通常要求 $\mathrm{SiC}_{\mathrm{f}} / \mathrm{SiC}$ 复合材料的界面层为片层状 结构, 以确保基体裂纹能够发生偏转, 增加材料的 韧性 ${ }^{[7]}$ 。片层状的热解炭 (PyC) 界面层是改善 $\mathrm{SiC}_{\mathrm{f}} / \mathrm{SiC}$ 复合材料力学性能的常用材料, 但其抗氧 化性较差, 在高温氧化环境中的应用受限 ${ }^{[8-9]}$ 。六方 晶体结构的氮化硼 $(\mathrm{h}-\mathrm{BN})$ 与 $\mathrm{PyC}$ 结构类似, 作为界 面层使用具有更优异的抗氧化性, $\mathrm{PyC}$ 的起始氧化 温度为 $450{ }^{\circ} \mathrm{C}, \mathrm{BN}$ 的起始氧化温度为 $800{ }^{\circ} \mathrm{C}^{[10-12]}$ 。 不同种类界面层交叠的 $(X / Y)_{n}$ 型复合界面层能够在 提高抗氧化性的同时减小界面层的厚度 ${ }^{[13]}$ 。在 $\mathrm{SiC}_{\mathrm{f}} / \mathrm{SiC}$ 复合材料中, $(\mathrm{PyC} / \mathrm{SiC})_{n}$ 和 $(\mathrm{BN} / \mathrm{SiC})_{n}$ 是最 为常用的复合界面体系。用 $\mathrm{SiC}$ 代替其中易氧化的 组分 $(\mathrm{PyC}$ 和 $\mathrm{BN})$, 可以在材料制备和服役过程中对 $\mathrm{PyC}$ 和 $\mathrm{BN}$ 界面层进行有效保护。Bertrand 等 ${ }^{[14-15]}$ 研究了 $(\mathrm{PyC} / \mathrm{SiC})_{n}$ 涂层对 Hi-Nicalon $/ \mathrm{SiC}$ 复合材料 性能的影响, 表明多层界面复合材料的高温力学性 能优于单层纤维涂层复合材料。 $\mathrm{YU}$ 等 ${ }^{[16]}$ 采用 $\mathrm{CVD}$ 法在 KD-I 碳化硅纤维上制备了 $(\mathrm{PyC} / \mathrm{SiC})_{n}$ 多层涂层, 采用 PIP 工艺制备三维 $\mathrm{KD}-\mathrm{I}-\mathrm{SiC}_{\mathrm{f}} / \mathrm{SiC}$ 复合材料, 结 果表明, $\mathrm{PyC} / \mathrm{SiC}$ 多层涂层能大大提高复合材料的 断裂韧性。MU 等 ${ }^{[17]}$ 以硼酸尿素和 PCS 为前驱体, 采用 PIP 工艺, 制备了 $\mathrm{BN} / \mathrm{SiC}$ 界面层, 双界面层复 合材料的拉伸性能和抗氧化性更加优异。在氧化环 境中, Hi-Nicalon/ $\left(\mathrm{BN}_{40}-\mathrm{SiC}_{25}\right)_{10} / \mathrm{SiC}$ 复合材料的使 用寿命明显提高 ${ }^{[18]}$ 。目前, 关于 $(\mathrm{PyC} / \mathrm{SiC})_{n}$ 界面层 研究较多, 对 $(\mathrm{BN} / \mathrm{SiC})_{n}$ 界面层研究较少, 同时采用 CVD 工艺制备 $\mathrm{SiC}$ 基体, 采用 PIP 工艺致密化的相 关报道很少。

一维 Mini 复合材料由沉积界面层的纤维束和 基体组成, 与宏观 $\mathrm{SiC}_{\mathrm{f}} / \mathrm{SiC}$ 复合材料的组成一致,
可以用来表征宏观复合材料基本尺度的结构和性 能。Mini 复合材料具有明显优点 ${ }^{[19]}$ : (1)制备周期短, 可以快速以较低的成本制备大量样品; (2)材料结构 简单, 能够表征材料的本征力学行为。因此本研究 采用 CVI 工艺在国产二代 $\mathrm{SiC}$ 纤维表面沉积 $\mathrm{BN}$ 和 $\mathrm{BN} / \mathrm{SiC}$ 复合界面层, 并以液态的乙烯基全氢聚碳硅 烷(VHPCS)为先驱体, 采用 PIP 工艺进行致密化, 制备 $\mathrm{Mini}-\mathrm{SiC}_{\mathrm{f}} / \mathrm{SiC}$ 复合材料。研究 VHPCS 的热解 产物、界面层的微观结构以及界面层种类对 $\mathrm{SiC}$ 纤 维及其 Mini 复合材料拉伸性能的影响, 并结合复合 材料的拉伸曲线和微观形貌, 研究界面层的多层化 对复合材料拉伸行为的影响。

\section{1 实验方法}

\section{1 制备纤维界面层}

实验中选用国防科技大学生产的 KD-II 型 $\mathrm{SiC}$ 纤维束 (规格 $1 \mathrm{~K}$ )作为增强体, 采用 CVI工艺制备界 面层、PIP 工艺制备基体。首先将 $\mathrm{SiC}$ 纤维用钼丝 束紧, 均匀坚直悬挂在 CVI 炉中间部位, 再以 $\mathrm{BCl}_{3}-\mathrm{NH}_{3}-\mathrm{H}_{2}-\mathrm{Ar}$ 为反应体系在 $\mathrm{SiC}$ 纤维表面沉积 $\mathrm{BN}$ 界面层, 沉积温度为 $900{ }^{\circ} \mathrm{C}, \mathrm{NH}_{3} / \mathrm{BCl}_{3}$ 物质量的 比为 $3 / 1, \mathrm{H}_{2} /\left(\mathrm{NH}_{3}+\mathrm{BCl}_{3}\right)$ 物质量的比为 $2 / 1, \mathrm{Ar}$ 作为 载气和稀释气, 沉积得到 $\mathrm{BN}$ 界面层; 以三氯甲基 硅烷(MTS)- $\mathrm{H}_{2}$ - $\mathrm{Ar}$ 为反应体系, $1100{ }^{\circ} \mathrm{C}$ 沉积 $\mathrm{SiC}$ 界 面层, $\mathrm{H}_{2} / \mathrm{MTS}$ 物质量的比为 $10 / 1$, 得到 $\mathrm{BN} / \mathrm{SiC}$ 界 面层。

\section{2 制备 Mini 复合材料}

沉积后的纤维束裁成 $25 \mathrm{~cm}$ 的长段, 以 VHPCS 为先驱体, 经过 PIP 循环五次制得具有不同界面层 的 $\mathrm{Mini}-\mathrm{SiC}_{\mathrm{f}} / \mathrm{SiC}$ 复合材料。PIP 工艺的热解采用分 段式热处理工艺: 第一段(室温 $250{ }^{\circ} \mathrm{C}$ ), 升温速率 $1{ }^{\circ} \mathrm{C} / \mathrm{min}$, 保温 $60 \mathrm{~min}$, 促进先驱体内部交联; 第二 段 $\left(250 \sim 550{ }^{\circ} \mathrm{C}\right)$, 升温速率 $1{ }^{\circ} \mathrm{C} / \mathrm{min}$, 保温 $60 \mathrm{~min}$, 防止热解速率过快形成较大的基体裂纹; 第三段 $\left(550 \sim 1100{ }^{\circ} \mathrm{C}\right)$, 升温速率 $5{ }^{\circ} \mathrm{C} / \mathrm{min}$, 保温 $60 \mathrm{~min}$ 。

在用 PIP 工艺制备复合材料的过程中, 将 $\mathrm{SiC}$ 基体引入 $\mathrm{SiC}$ 纤维中使其致密化。对于一维的 Mini 复合材料, 线密度可以作为考察致密化程度的指标, 浸渍不同次数后复合材料的线密度结果如表 1 所示。 
表 1 浸渍不同次数后 Mini 复合材料的线密度

Table 1 The linear density of Mini-composites after different PIP cycles

\begin{tabular}{ccccccc}
\hline \\
Lample
\end{tabular}

采用 CVI 工艺沉积界面层, 得到了三种不同线密度 的 $\mathrm{SiC}$ 纤维束, 纤维的线密度增加。浸渍初期, 由于 纤维中基体引入较多, 复合材料的线密度明显增大, 当进行第二次至第五次浸渍后, 线密度增大的幅度 基本平稳，趋于稳定。

\section{3 测试表征}

利用称重法测试计算 Mini 复合材料的线密度。 采用日本理学株式会社 $\mathrm{D} / \mathrm{max}-\mathrm{RB}$ 型 $\mathrm{X}$ 射线衍射仪 分析界面层和 VHPCS 裂解产物的物相组成和晶体 结构。测试条件为铜靶、 $\mathrm{K} \alpha$ 射线，加速电压 $40 \mathrm{kV}$ 、 电流 $50 \mathrm{~mA}, 2 \theta=10^{\circ} \sim 90^{\circ}$ 。采用美国 INSTRON5944 型拉伸试验机测试单丝纤维的强度, 测试样品 25 个。采用美国 INSTRON 5567 型万能拉伸试验机测 试 Mini- $\mathrm{SiC}_{\mathrm{f}} / \mathrm{SiC}$ 复合材料的拉伸性能, 加载速度为 $2.0 \mathrm{~mm} / \mathrm{min}$ 。采用日立 Hitachi S4800 型扫描电镜表 征界面层和 Mini 复合材料的微观形貌。

\section{2 结果与讨论}

\section{1 界面层的微观结构}

用 SEM 和 EDS 分析了不同纤维表面和断面的 微观形貌和元素组成(图 1)。原纤维表面光滑、均匀 致密，没有明显晶粒堆积; 沉积了 $\mathrm{BN}$ 界面层纤维 仍然保留了较为光滑致密的表面，与界面层结合紧 密，避免界面层从纤维表面脱落。BN 界面层的 EDS 分析结果显示含有 $\mathrm{B} 、 \mathrm{~N} 、 \mathrm{O} 、 \mathrm{C}$ 和 $\mathrm{Si}$ 元素, 其中的 $\mathrm{O}$ 元素是由于 $\mathrm{BN}$ 结晶性较差, 反应活性较高, 与空 气中 $\mathrm{H}_{2} \mathrm{O}$ 或 $\mathrm{O}_{2}$ 反应产生的 ${ }^{[20]}$; 由沉积 $\mathrm{BN} / \mathrm{SiC}$ 界面 层的纤维可以看出, CVI 工艺制备的 $\mathrm{SiC}$ 界面层表 面分布着细小的颗粒, 呈我鸟卵石状紧密堆积。BN 与 $\mathrm{SiC}$ 界面层结合紧密, 界面层之间特征明显, 从放 大图中可以观察到明显的 $\mathrm{BN}$ 和 $\mathrm{SiC}$ 界面分界, $\mathrm{SiC}$ 界面层的 $\mathrm{EDS}$ 分析结果主要为 $\mathrm{C}$ 和 $\mathrm{Si}$ 元素, 含有 微量的 $\mathrm{O}$ 。

图 2 为纤维表面 $\mathrm{BN}$ 界面层和 $\mathrm{BN} / \mathrm{SiC}$ 复合界面 层的 XRD 图谱, 从图中可以看出, 沉积后的 $\mathrm{BN}$ 界 面层在 $2 \theta=26.2^{\circ}$ 和 $43.1^{\circ}$ 处观察到两个较宽的衍射峰,
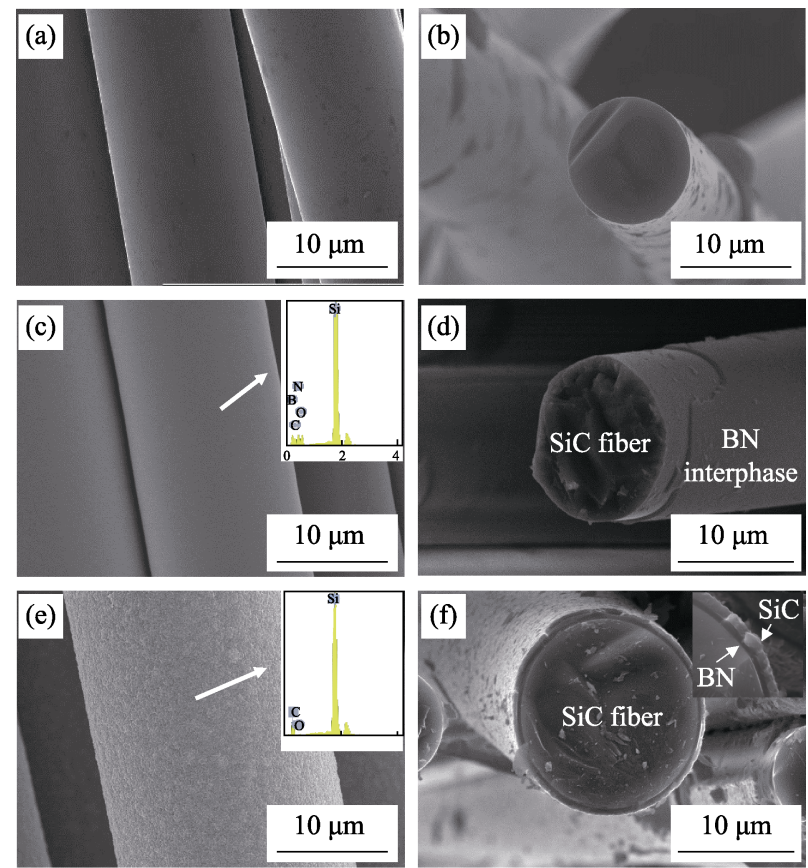

图 1 不同种类界面层纤维的 SEM 照片及 EDS 分析结果

Fig. 1 SEM and EDS microstructures of fibers with different interphases

(a, b) As-received; (c, d) BN-coated; (e, f) BN/SiC-coated

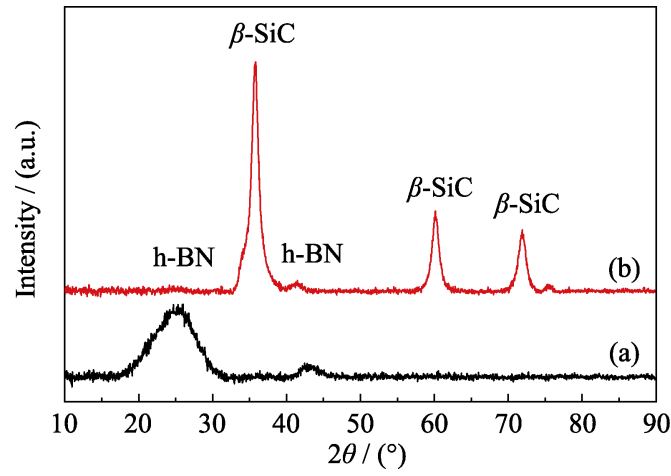

图 $2 \mathrm{BN}(\mathrm{a})$ 和 $\mathrm{BN} / \mathrm{SiC}(\mathrm{b})$ 界面层的 XRD 图谱

Fig. 2 XRD patterns of $\mathrm{BN}$ (a) and $\mathrm{BN} / \mathrm{SiC}$ (b) interphase

归属于 h-BN 的(002)和(101)晶面, 说明沉积的 BN 界面层中存在六方相，但是其晶体尺寸较小，衍射 峰较宽; 沉积的 $\mathrm{BN} / \mathrm{SiC}$ 复合界面层在 $2 \theta=35.5^{\circ}$ 、 $60.0^{\circ}$ 和 $71.5^{\circ}$ 处可观察到分别归属于 $\beta-\mathrm{SiC}$ 的(111)、 (220)和(311)的衍射峰; 与 $\mathrm{BN}$ 的衍射峰相比, $\mathrm{SiC}$ 的 
衍射峰强度相对较大, 峰形尖锐, 表明 $\mathrm{SiC}$ 晶粒尺 寸较大, 晶型结构完整。

晶粒尺寸 $(<100 \mathrm{~nm})$ 可以采用 Scherrer 公式进行 计算:

$$
D=\frac{K \lambda}{\beta \cos \theta}
$$

式中, $D$ 为平均晶粒尺寸, $\theta$ 为散射角, $\lambda$ 为 $\mathrm{X}$ 射线波 长 $(0.15406 \mathrm{~nm}), \beta$ 为衍射峰的半高宽, $K$ 为 Scherrer 常数(0.89)。根据式(1)计算的沉积 $\mathrm{BN}$ 和 $\mathrm{SiC}$ 界面层 晶粒尺寸分别为 1.76 和 $18.73 \mathrm{~nm}$ 。从计算的晶粒尺 寸可知, BN 界面层中虽然存在 h-BN, 但是其晶粒 尺寸较小; $\mathrm{SiC}$ 界面层的晶粒尺寸远大于 $\mathrm{BN}$ 界面层 的晶粒尺寸。

图 3 为 $\mathrm{BN}$ 界面层和 $\mathrm{SiC}$ 界面层的 HRTEM 照 片。由图 3(a) 可以看出, BN 界面层中存在随机取向 的六方晶体结构, 其晶面间距较大 $(d=0.334 \mathrm{~nm})$, 晶 粒尺寸较小, 为浴轮层状结构, 表明 $\mathrm{BN}$ 层缺乏三 维有序性, 纳米尺寸的 BN 晶粒在界面层中多向分 散。由 $\mathrm{SiC}$ 界面层(图 3(b)) 的 HRTEM 照片可以看 出, 界面层中具有尺寸较大的 $\mathrm{SiC}$ 晶粒, 大部分为 结晶状态, 晶面间距为 $0.251 \mathrm{~nm}$, 对应于 $\beta-\mathrm{SiC}$ 的 (111)晶面, 其晶粒尺寸远大于 $\mathrm{BN}$ 界面层中 h-BN 的晶粒尺寸。

\section{2 界面层对 $\mathrm{SiC}$ 纤维性能的影响}

图 4 为沉积界面层后纤维的拉伸强度。沉积界 面层后纤维的拉伸强度有所降低, $\mathrm{BN}$ 界面层和 $\mathrm{SiC}$ 界面层的纤维抗拉强度分别为 2.39 和 $2.11 \mathrm{GPa}$, 强 度保留率分别为 $93.7 \%$ 和 $82.6 \%$ 。

纤维的单丝拉伸强度可以根据式(2)计算:

$$
\sigma=\frac{4 P_{\max }}{\pi D^{2}}
$$

式中, $\sigma 、 P_{\max }$ 和 $D$ 分别为拉伸强度、最大载荷和纤 维直径。从式中可以看出, 纤维的拉伸强度与直径 的平方成反比。因此, 沉积界面层后, 纤维拉伸强度 的降低, 可能是由于纤维的体积效应。
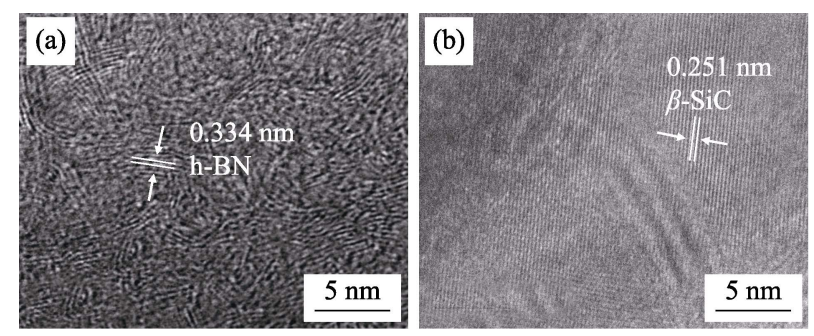

图 3 不同界面层的 HRTEM 照片

Fig. 3 HRTEM microstructures of different interphases (a) $\mathrm{BN}$ interphase; (b) $\mathrm{SiC}$ interphase

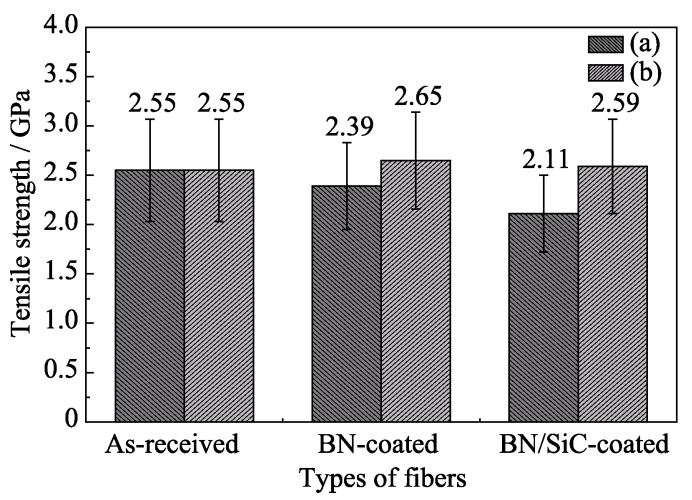

图 4 不同种类界面层纤维的拉伸强度

Fig. 4 Tensile strength of SiC fibers with different interphases (a) Tested strength value; (b) Calculated strength value after deducting the thickness of interphases

分析单丝拉伸测试结果发现, 原纤维、沉积 $\mathrm{BN}$ 和 $\mathrm{BN} / \mathrm{SiC}$ 纤维中 25 个样品的平均直径分别为 12.23、12.87 和 $13.56 \mu \mathrm{m}$, 据此可以计算出界面层 的厚度, $\mathrm{BN}$ 约为 $320 \mathrm{~nm}, \mathrm{SiC}$ 约为 $345 \mathrm{~nm}$ 。为表征 纤维本征的拉伸强度, 根据式(2), 扣除界面层厚度 的影响, 按照原纤维直径 $12.23 \mu \mathrm{m}$ 进行计算, 纤维 的拉伸强度分别为 2.65 和 $2.59 \mathrm{GPa}$, 与原纤维 $(2.55 \mathrm{GPa})$ 相比略有提高。这是因为 $\mathrm{SiC}$ 纤维是一种 脆性材料, 其拉伸强度与纤维中裂纹和缺陷的数量 密切相关 ${ }^{[19]}$, 界面层能够在一定程度上弥合纤维表 面的裂纹, 从而提高拉伸强度。

沉积界面层后纤维单丝的弹性模量与原纤维相 当, 基本保持不变, 如图 5 所示。这是因为弹性模量 是 $\mathrm{SiC}$ 纤维固有的性质, 反映的是原子间平均健强, 与纤维的结构、晶粒尺寸以及组成等密切相关 ${ }^{[20]}$ 。 BN 和 $\mathrm{SiC}$ 界面层的沉积温度不超过 $1100{ }^{\circ} \mathrm{C}$, 低于 $\mathrm{SiC}$ 纤维制备过程中的热处理温度, 不会造成纤维结 构、晶体尺寸和 $\mathrm{SiC}$ 原子间距的变化, 从而未引起 弹性模量的变化。

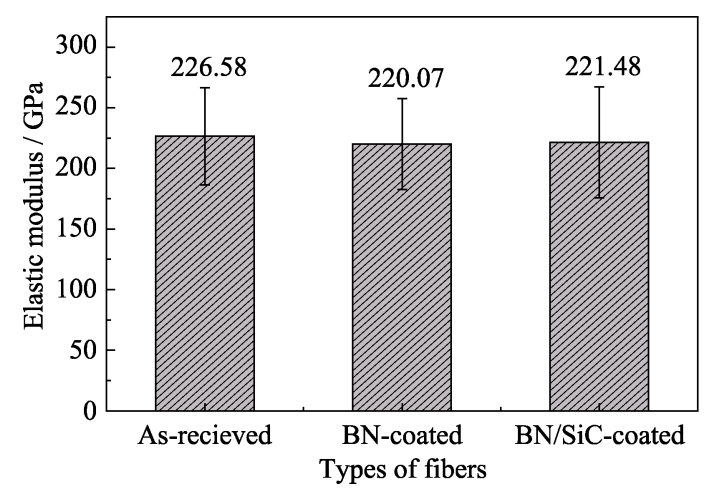

图 5 不同种类界面层纤维的弹性模量

Fig. 5 Elastic modulus of $\mathrm{SiC}$ fibers with different interphases 


\subsection{Mini 复合材料拉伸力学行为}

图 6 为 VHPCS 经过 $1100{ }^{\circ} \mathrm{C}$ 热解后粉末的 $\mathrm{XRD}$ 图谱。从图中可以看出, 在 $2 \theta=35.6^{\circ} 、 60.1^{\circ}$ 和 $71.7^{\circ}$ 处存在衍射峰, 分别归属于 $\beta-\mathrm{SiC}$ 的(111)、 (220)和(311)晶面; 在 $2 \theta=26.5^{\circ}$ 处存在衍射峰, 归 属于石墨结构碳的 (002) 晶面, 说明热解形成的陶瓷 中存在少量的自由碳, 为石墨微型结构, 热解产物中 碳硅比大于 1 。根据谢乐公式计算 VHPCS 在 $1100{ }^{\circ} \mathrm{C}$ 热解后 $\mathrm{SiC}$ 的晶粒尺寸为 $6.89 \mathrm{~nm}$ 。

图 7 为 $\mathrm{Mini}-\mathrm{SiC}_{\mathrm{f}} / \mathrm{SiC}$ 复合材料的 $\mathrm{SEM}$ 照片, 可 以看出, 采用 PIP 工艺制备的 $\mathrm{Mini}-\mathrm{SiC}_{\mathrm{f}} / \mathrm{SiC}$ 复合材 料结构致密, 纤维分布均匀, 经过五次浸渍后基体 内部虽然存在少量的裂纹, 但已经彼此连接堵塞孔 道, 难以进一步提升致密度。由图 7(a)可知, 无界面 层的复合材料断口处虽然存在少量纤维拔出形成的 孔洞，但是多数纤维与基体齐平断裂，纤维的增韧 作用并未得到发挥。BN 界面层复合材料断裂面如 图 7(b)所示, 断口处纤维拔出的长度和形成的孔洞 明显增多，纤维和基体间脱粘且表面粗粘，说明界 面层在复合材料拉伸断裂过程中起到了增强作用。类 似的现象也在图 7(c)BN/SiC 界面层复合材料断口处 观察到。断面纤维的表面有少量的界面层碎片, 多 数拔出纤维的表面光滑。

图 8 为三种 Mini 复合材料的载荷应变曲线。由 图 8 可知, 无界面层的 $\mathrm{SiC}_{\mathrm{f}} / \mathrm{SiC} \mathrm{Mini}$ 复合材料最大 载荷和应变分别为 $133.13 \mathrm{~N}$ 和 $0.405 \% 。 \mathrm{SiC}_{\mathrm{f}} / \mathrm{BN} / \mathrm{SiC}$ 和 $\mathrm{SiC}_{\mathrm{f}} /(\mathrm{BN} / \mathrm{SiC}) / \mathrm{SiC}$ Mini 复合材料的最大载荷和应 变分别达到 $228.43 \mathrm{~N}$ 和 $0.64 \%$ 、235.7 N 和 $0.701 \%$ 。 从图中可以看出, 无界面层复合材料断裂应变较小, 可承受载荷小; 而有界面层的复合材料, 断裂应变 明显增大，断裂延伸率增大表明复合材料断裂时消 耗的能量增加, 可承受的载荷增大。有界面层的复 合材料无论是最大载荷还是应变均相差不大。对 $\mathrm{SiC}_{\mathrm{f}} / \mathrm{SiC}$ 复合材料, 基体 $\mathrm{SiC}$ 的断裂应变比纤维小, 因此复合材料受拉应力时裂纹通常先在基体中产生, 后扩展至纤维表面。

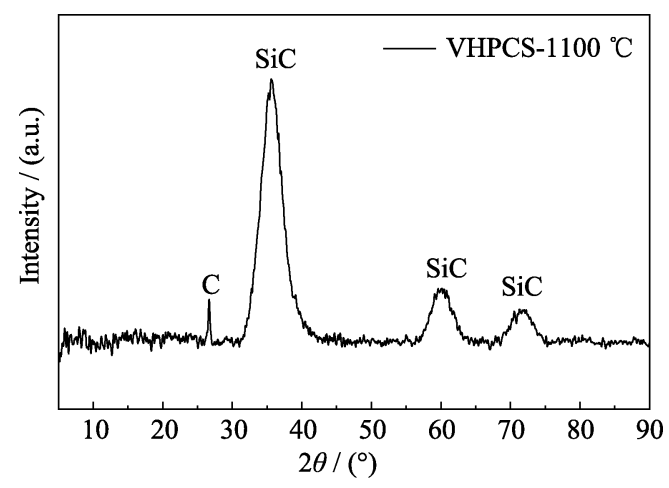

图 6 VHPCS-1100 ${ }^{\circ} \mathrm{C}$ 热解产物的 XRD 图谱

Fig. 6 XRD pattern of the pyrolysis products from VHPCS at $1100{ }^{\circ} \mathrm{C}$

由 XRD 和 TEM 分析结果可以看出, BN 界面层 中含有少量 h-BN 微晶, 作为内界面层使用, 能够对 纤维表面进行修饰, 发挥纤维的增强增韧作用 ${ }^{[21]}$ 。但 是由于 $\mathrm{BN}$ 结晶度不高, 增㓞效果有限, 需要通过 后续工艺优化制备接近理想的 h-BN。沉积的 $\mathrm{SiC}$ 为 高结晶的晶体结构, 由于其不是片层结构, 基体产 生的裂纹传递到 $\mathrm{SiC}$ 界面层时脆性断裂, 裂纹偏转 作用不明显, 直接扩展至 $\mathrm{BN}$ 界面层。因此起主要 裂纹偏转作用的为 $\mathrm{BN}$ 界面层, 是 $\mathrm{SiC}_{\mathrm{f}} / \mathrm{BN} / \mathrm{SiC}$ 和 $\mathrm{SiC}_{\mathrm{f}} /(\mathrm{BN} / \mathrm{SiC}) / \mathrm{SiC}$ 复合材料所能承受的拉伸应变和 最大载荷基本一致的主要原因, 表明 $\mathrm{BN}$ 界面层的 裂纹偏转作用可以提高复合材料的力学性能 ${ }^{[22]}$ 。

\section{3 结论}

采用 CVI 工艺制得均匀致密的 BN 界面层及其 $\mathrm{BN} / \mathrm{SiC}$ 复合界面层, 研究了界面层种类对 $\mathrm{SiC}$ 纤维 和 Mini 复合材料性能的影响:

1) BN 界面层中存在晶粒尺寸较小 $(1.76 \mathrm{~nm})$ 的六 方相; $\mathrm{SiC}$ 界面层结晶性较好, 晶粒尺寸为 $18.73 \mathrm{~nm}$ 。

2) 沉积 $\mathrm{BN}$ 和 $\mathrm{BN} / \mathrm{SiC}$ 界面层后, $\mathrm{SiC}$ 纤维的弹 性模量基本不变, 拉伸强度降低, 保留率分别为 93.3\%和 $82.6 \%$; 扣除界面层厚度的影响, 沉积界面
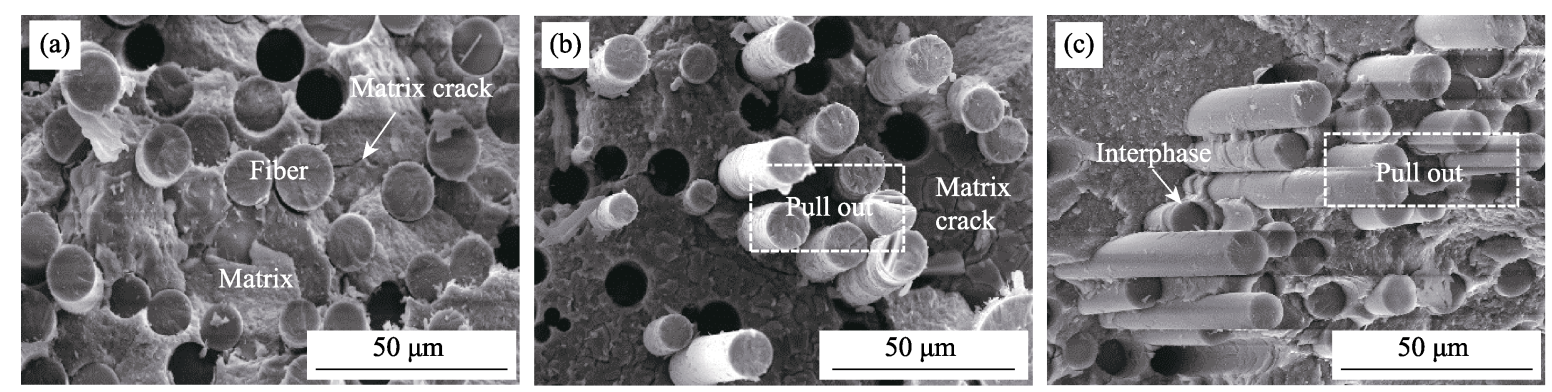

图 7 不同界面层复合材料断口的 SEM 照片

Fig. 7 Cross-section morphologies of $\operatorname{mini}-\mathrm{SiC}_{\mathrm{f}} / \mathrm{SiC}$ composites with different interphases (a) No interphase, (b) BN interphase, (c) BN/SiC interphase 


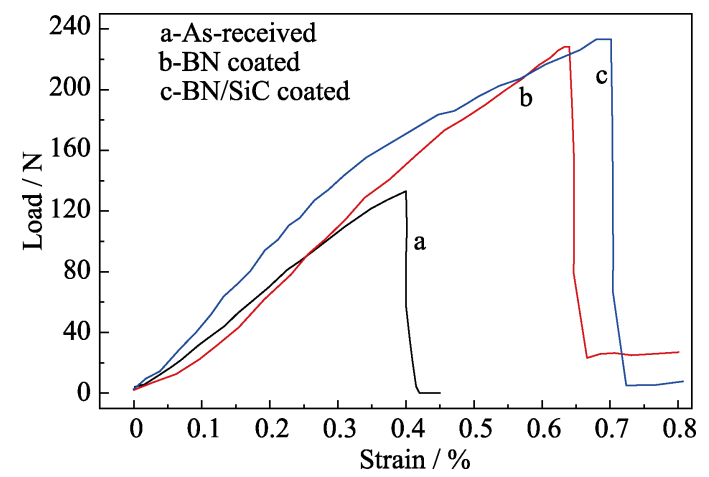

图 $8 \quad \mathrm{Mini}^{-} \mathrm{SiC}_{\mathrm{f}} / \mathrm{SiC}$ 复合材料载荷应变曲线

Fig. 8 The load-strain curves of $\mathrm{Mini}-\mathrm{SiC}_{\mathrm{f}} / \mathrm{SiC}$ composites

层的纤维拉伸强度分别为 2.65 和 $2.59 \mathrm{GPa}$, 与原纤 维 $(2.55 \mathrm{GPa})$ 相近;

3) $\mathrm{SiC}_{\mathrm{f}} / \mathrm{SiC} 、 \mathrm{SiC}_{\mathrm{f}} / \mathrm{BN} / \mathrm{SiC} 、 \mathrm{SiC}_{\mathrm{f}} /(\mathrm{BN} / \mathrm{SiC}) / \mathrm{SiC}$ 三 种 Mini复合材料的最大拉伸载荷分别为 $133.13 、 228.43$ 和 $235.7 \mathrm{~N}$, 最终拉伸应变分别为 $0.405 \% 、 0.640 \%$ 和 $0.701 \%$; $\mathrm{BN}$ 和 $\mathrm{BN} / \mathrm{SiC}$ 界面层可以提高复合材料 的力学性能, 其中 $\mathrm{BN}$ 界面层在材料断裂过程中起 主要的裂纹偏转作用。

\section{参考文献:}

[1] NASIRI N A, PATRA N, NI N, et al. Oxidation behaviour of $\mathrm{SiC} / \mathrm{SiC}$ ceramic matrix composites in air. Journal of the European Ceramic Society, 2016, 36(14): 3293-3302.

[2] 刘虎, 杨金华, 周怡然, 等. 国外航空发动机用 $\mathrm{SiC}_{\mathrm{f}} / \mathrm{SiC}$ 复合材 料的材料级性能测试研究进展. 材料工程, 2018, 46(11): 1-12.

[3] STAEHLER J M, ZAWADA L P. Performance of four ceramic-matrix composite divergent flap inserts following ground testing on an F110 turbofan engine. Journal of the American Ceramic Society, 2000, 83(7): 1727-1738.

[4] 焦健, 陈明伟. 新一代发动机高温材料-陶瓷基复合材料的制 备、性能及应用. 航空制造技术, 2014, 451(7): 62-69.

[5] CHARLES H, HENAGER J, KURTZ R J. Low-activation joining of $\mathrm{SiC} / \mathrm{SiC}$ composites for fusion applications. Journal of Nuclear Materials, 2011, 417(1/2/3): 375-378.

[6] LAMON J. A micromechanics-based approach to the mechanical behavior of brittle matrix composites. Composites Science Technology, 2001, 61(15): 2259-2272.

[7] REBILLAT F, LAMON J, NASLAIN R, et al. Interfacial bond strength in $\mathrm{SiC} / \mathrm{C} / \mathrm{SiC}$ composite materials, as studied by single-fibre push-out tests. Journal of American Ceramic Society, 1998, 81(4): 965-978.

[8] SREEJITH K, VIPIN V, SUBRAMANIA S, et al. A comparative

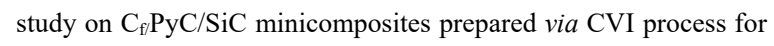

hypersonic engine application. International Journal of Applied Ceramic Technology, 2018, 15(5): 1110-1123.

[9] WINDISCH C F, HENAGER C, SPRINGER G D, et al. Oxidation of the carbon interface in Nicalon fiber reinforced silicon carbide composite. Journal of the American Ceramic Society, 1997, 80(3): 569-574.

[10] SHI YING, ARAKI HIROSHI, YANG WEN, et al. Influence of fiber pre-coating on mechanical properties and interfacial structures of $\mathrm{SiC}(\mathrm{f}) / \mathrm{SiC}$ composites. Journal of Inorganic Materials, 2001, 16(5): $883-888$.

[11] OPILA E J, VERRILLI M J. Borosilicate glass-induced fiber degradation of $\mathrm{SiC} / \mathrm{BN} / \mathrm{SiC}$ composites exposed in combustion environments. International Journal of Applied Ceramic Technology, 2016, 13(3): 434-442.

[12] WING B L, HALLORAN J W. Subsurface oxidation of boron nitride coatings on silicon carbide fibers in $\mathrm{SiC} / \mathrm{SiC}$ ceramic matrix composites. Ceramics International, 2018, 44(14): 17499-17505.

[13] NASLAIN R R, PAILLER R J F, LAMON J L. Single and multilayered interphases in $\mathrm{SiC} / \mathrm{SiC}$ composites exposed to severe environmental conditions: an overview. International Journal of Applied Ceramic Technology, 2010, 7(3): 263-275.

[14] BERTRAND S, FORIO P, PAILLER R, et al. Hi-Nicalon/SiC minicomposites with (pyrocarbon $/ \mathrm{SiC})_{n}$ nanoscale multilayered interphases. Journal of the American Ceramic Society, 1999, 82(9): 2465-2473.

[15] BERTRAND S, PAILLER R, LAMON J. SiC/SiC minicomposites with nanoscale multilayered fibre coatings. Composites Science and Technology, 2001, 61(3): 363-367.

[16] YU HAIJIAO, ZHOU XINGUI, ZHANG WEI, et al. Mechanical behavior of $\mathrm{SiC}_{\mathrm{f}} / \mathrm{SiC}$ composites with alternating $\mathrm{PyC} / \mathrm{SiC}$ multilayer interphases. Materials and Design, 2013, 44: 320-324.

[17] YANG M, ZHOU W C, LUO F, et al. Effects of BN/SiC dual-layer interphase on mechanical and dielectric properties of $\mathrm{SiC}_{\mathrm{f}} / \mathrm{SiC}$ composites. Ceramic International, 2014, 40(2): 3411-3418.

[18] BERTRAND S, BOISRON O, PAILLER R, et al. (PyC-SiC) ${ }_{n}$ and $(\mathrm{BN}-\mathrm{SiC})_{n}$ nano-scale multilayered interphases by pressure pulsed-CVI. Key Engineering Materials, 1999, 164-165: 357-360.

[19] AMJAD A, EMMANUEL M, SIVAKUMAR R, et al. Effect of fiber content on single tow $\mathrm{SiC}$ minicomposite mechanical and damage properties using acoustic emission. Journal of the European Ceramic Society, 2015, 35(13): 3389-3399.

[20] LV X X, QI Z, JIANG Z Y, et al. The Microstructure and Mechanical Properties of Silicon Carbide Fibers with Boron Nitride Interphase. IOP Conf. Series: Materials Science and Engineering, 2019, 678, 012061.

[21] WANG GUODONG, SONG YONGCAI. Enhancing mechanical property of $\mathrm{SiC}$ fiber by decreasing fiber diameter through a modified melt-spinning process. Journal of Inorganic Materials, 2018, 33(7): 721-727.

[22] MU Y, ZHOU W C, WANG C, et al. Mechanical and electromagnetic shielding properties of $\mathrm{SiC}_{\mathrm{f}} / \mathrm{SiC}$ composites fabricated by combined CVI and PIP process. Ceramics International, 2014, 40(7): 10037-10041. 